\title{
Contact Hamiltonian Description of Systems with Exponentially Decreasing Force and Friction that is Quadratic in Velocity
}

\author{
Furkan Semih Dündar ${ }^{1}$ \\ ${ }^{1}$ Department of Mechanical Engineering, Faculty of Technology, Amasya University, Amasya, Turkey
}

\section{Article Info}

Keywords: Contact geometry, Contact Hamiltonian, Friction 2010 AMS: 53D10, 70G45

Received: 8 April 2020

Accepted: 21 May 2020

Available online: 10 June 2020

\begin{abstract}
We have given a simple contact Hamiltonian description of a system with exponentially vanishing (or zero) potential under a friction term that is quadratic in velocity. We have given two applications: to cavity solitons and to a free body under air friction.
\end{abstract}

\section{Introduction}

Hamiltonian mechanics is done in phase space which is a symplectic manifold. Symplectic mainfolds are even dimensional mainfolds equipped with a symplectic 2-form $\omega$. On the other hand, contact geometry is carried on odd dimensional manifolds equipped with a contact 1-form $\eta$. $\eta$ is a contact form if $\eta \wedge d \eta \wedge \cdots \wedge d \eta \neq 0$ (in a $2 n+1$ dimensional contact manifold, the term $d \eta$ is wedge-multiplied $n$ times). For more information on contact manifolds, the reader is referred to [1] and to [2] for the relation between contact geometry and the Huygens' Principle.

Recently, Hamiltonian mechanics has been generalized to work in contact manifolds with the addition of extra parameter $S$ [3]. Ref. [4] studied variational aspects of contact Hamiltonian mechanics and ref. [5] applied contact geometric methods to a theory of gravity called shape dynamics [6-8] (see ref. [9] for a review of shape dynamics). Also in ref. [3] time-dependent contact Hamiltonians are introduced. In our study, we will use time-independent contact Hamiltonian mechanics in 1D. So the variables we have are $q, p, S$. In this variables the equations of motion derived from the contact Hamiltonian $(H)$ read as [3]:

$$
\begin{aligned}
\dot{q} & =\frac{\partial H}{\partial p} \\
\dot{p} & =-\frac{\partial H}{\partial q}-p \frac{\partial H}{\partial S} \\
\dot{S} & =p \frac{\partial H}{\partial p}-H .
\end{aligned}
$$

The organization of the paper is as follows: in Section 2 we introduce the contact Hamiltonian we use, in Section 3 we give a solution of the equations of motion derived from the contact system, in Section 4 we give two applications where our description can be used and finally in Section 5 we conclude the paper. 


\section{Contact Hamiltonian description}

It is known in the literature (see ref. [3]) that the contact Hamiltonian $H=p^{2} / 2 m+V(q)+\gamma S$ describes a system with frictional force linear in velocity for an arbitrary potential term $V(q)$. In this study we make a minor change in the last term and use the following contact Hamiltonian:

$$
H=\frac{p^{2}}{2 m}+V(q)+\gamma p S
$$

The equations of motion that follow are as:

$$
\begin{aligned}
\dot{q} & =\frac{p}{m}+\gamma S, \\
\dot{p} & =-V^{\prime}(q)-\gamma p^{2}, \\
\dot{S} & =\frac{p^{2}}{2 m}-V(q) .
\end{aligned}
$$

When we take the time derivative of Equation (2.1) we obtain:

$$
\begin{aligned}
\ddot{q} & =\frac{\dot{p}}{m}+\gamma \dot{S}, \\
& =-\frac{1}{m}\left(V^{\prime}(q)+\gamma p^{2}\right)+\gamma\left(\frac{p^{2}}{2 m}-V(q)\right), \\
m \ddot{q} & =-V^{\prime}(q)-\frac{\gamma}{2} p^{2}-m \gamma V(q) .
\end{aligned}
$$

Using Equation (2.1) we can write $p=m \dot{q}-m \gamma S$. As an ansatz let us assume $S=\alpha \dot{q}$ for some $\alpha$. Then we get $p=m(1-\gamma \alpha) \dot{q}$. If we put this form of $p$ into Equation (2.4) we obtain:

$$
m \ddot{q}+\frac{m^{2} \gamma}{2}(1-\gamma \alpha)^{2} \dot{q}^{2}=-V^{\prime}(q)-m \gamma V(q)
$$

On the other hand, when we use the ansatz $S=\alpha \dot{q}$ in Equation (2.3) we obtain:

$$
\alpha \ddot{q}=\dot{S}=\frac{m}{2}(1-\gamma \alpha)^{2} \dot{q}^{2}-V(q)
$$

In order to be consistent, Equation (2.5) and Equation (2.6) must give the same answer. So we must have the following:

$$
\frac{m^{2}}{2 \alpha}(1-\gamma \alpha)^{2} \dot{q}^{2}-\frac{m}{\alpha} V(q)=-\frac{m^{2} \gamma}{2}(1-\gamma \alpha)^{2} \dot{q}^{2}-V^{\prime}(q)-m \gamma V(q)
$$

Equating the terms in front of $\dot{q}^{2}$ on both sides gives us $\alpha=-1 / \gamma$. There appears a condition on the potential $V(q)$ :

$$
V^{\prime}(q)=-2 m \gamma V(q)
$$

So with the ansatz we put, arbitrary potentials are not allowed. The solution of Equation (2.7) is elementary:

$$
V(q)=A \exp (-2 m \gamma q)
$$

for some constant $A$. Now we have determined $\alpha$ in $S=\alpha \dot{q}$ as $\alpha=-1 / \gamma$. As a consistency check let us put this in Equation (2.1) and obtain $p=2 m \dot{q}$. On the other hand we have $\dot{p}=-V^{\prime}(q)-\gamma p^{2}$ from Equation (2.2). This yields: 


$$
2 m \ddot{q}=-\frac{\partial}{\partial q}(A \exp (-2 m \gamma q))-\gamma p^{2}
$$

and

$$
m \ddot{q}+2 \gamma m^{2} \dot{q}^{2}=A m \gamma \exp (-2 m \gamma q) .
$$

Comparing this Equation with Equation (2.6) we see that there is no inconsistency. So the consistent equation of motion is as follows:

$$
m \ddot{q}+\gamma_{n} \dot{q}^{2}=-\frac{\partial}{\partial q} A_{n} e^{-\gamma_{n} q / m}
$$

where $\gamma_{n}$ (new $\gamma$ ) is given through $\gamma_{n}=2 m^{2} \gamma$ and $A_{n}$ (new $A$ ) is $A_{n}=A / 2$.

\section{Solution of the equation of motion}

In this Section, we will solve the equation of motion of the system given by Equation (2.8). Let us define $Q=\gamma_{n} q / m$. So we have:

$$
\ddot{Q}+\dot{Q}^{2}=\frac{A_{n} \gamma_{n}^{2}}{m^{3}} e^{-Q}
$$

We now define $F$ via $Q=\log F$. Then we get:

$$
\frac{\ddot{F}}{F}-\frac{\dot{F}^{2}}{F^{2}}+\frac{\dot{F}^{2}}{F^{2}}=\frac{A_{n} \gamma_{n}^{2}}{m^{3}} \frac{1}{F}
$$

The second and third terms cancel out with each other and we obtain $\ddot{F}=A_{n} \gamma_{n}^{2} / \mathrm{m}^{3}$. Making changes of variables in the reverse order, one obtains:

$$
q(t)=\frac{m}{\gamma_{n}} \log \left(\frac{A_{n} \gamma_{n}^{2}}{2 m^{3}} t^{2}+c_{1} t+c_{2}\right)
$$

where $c_{1}$ and $c_{2}$ are two constants of integration.

\section{Possible applications}

The equation of motion (see Equation (2.8)) derived from the contact Hamiltonian, $H=p^{2} / 2 m+V(q)+\left(\gamma_{n} / 2 m^{2}\right) p S$, is:

$$
m \ddot{q}+\gamma_{n} \dot{q}^{2}=-\frac{\partial}{\partial q} A_{n} e^{-\gamma_{n} q / m}
$$

where $\gamma_{n}, A_{n}$ are some constant parameters. In this Section, we give two possible applications of our choice of contact Hamiltonian. The first one is cavity solitons with friction quadratic in velocity, and the other one is air friction with quadratic dependency on velocity.

\subsection{Cavity solitons}

Recently Ref. [10] put forward that cavity solitons (for a review see Ref. [11]) may be modeled with an effective potential of the form $-K^{2} e^{-r / R}$ with the strength $K$ and the range $R$. Our potential term is $V(q)=A_{n} e^{-\gamma_{n} q / m}$. So if we choose $A=-K^{2}$ and $\gamma_{n}=m / R$ we can model cavity solitons. But our model has a quadratic friction term: $\gamma_{n} \dot{q}^{2}=(m / R) \dot{q}^{2}$. It may be possible that our contact Hamiltonian can model cavity solitons with an exponentially decreasing force and a friction term that is quadratic in velocity. 


\subsection{Air friction}

It is well known in literature that for large bodies, air friction can be modelled with frictional force that has quadratic dependency on the velocity. Therefore our contact Hamiltonian may also model a free particle under air friction if $A_{n}=0$ or with a driving force equal to $\left(A_{n} \gamma_{n} / m\right) e^{-\gamma_{n} q / m}$.

\section{Conclusion}

Recently contact Hamiltonian mechanics has gained some interest [3-5]. In this paper we used a simple contact Hamiltonian to account for quadratic dependence on velocity. As we mentioned in Section 4 this description may be useful for modelling cavity solitons with a quadratic friction term or air friction for free particles. We note that our work is only an initial step towards giving a contact Hamiltonian description of a system with an arbitrary potential under a friction that is quadratic in velocity.

\section{Acknowledgements}

We would like to thank Metin Arık and Bayram Tekin for useful discussions. We also would like to Gülhan Ayar for bringing contact geometry to our attention.

\section{References}

[1] H. Geiges, A brief history of contact geometry and topology, Expo. Math., 19(1) (2001), 25-53.

[2] H. Geiges, Christiaan huygens and contact geometry, (2005) arXiv:math/0501255.

[3] A. Bravetti, H. Cruz, D. Tapias, Contact Hamiltonian mechanics, Ann. Phys.-New York, 376 (2017), 17-39.

[4] Q. Liu, P. J. Torres, C. Wang, Contact Hamiltonian dynamics: Variational principles, invariants, completeness and periodic behavior, Ann. Phys.-New York, 395 (2018), 26-44

[5] D. Sloan, Dynamical similarity. Phys. Rev. D, 97(12) (2018), 123541.

[6] E. Anderson, J. Barbour, B. Foster, N. Ó Murchadha, Scale-invariant gravity: Geometrodynamics. Classical Quant. Grav., 20 (2003), $1571-1604$.

[7] E. Anderson, J. Barbour, B. Z. Foster, B. Kelleher, N. Ó. Murchadha, The physical gravitational degrees of freedom, Classical Quant. Grav., 22 (2005), $1795-1802$.

[8] J. Barbour, N. O Murchadha, Classical and Quantum Gravity on Conformal Superspace, (1999), arXiv:gr-qc/9911071.

[9] F. Mercati, A Shape Dynamics Tutorial, (2014), arXiv:1409.0105.

[10] S. R. Anbardan, C. Rimoldi, R. Kheradmand, G. Tissoni, F. Prati, Exponentially decaying interaction potential of cavity solitons, Phys. Rev. E, 97(3) (2018), 032208.

[11] L. A. Lugiato, F. Prati, M. Brambilla, L. Columbo, S. Barland, G. Tissoni, K. M. Aghdami, R. Kheradmand, H. Tajalli, H. Vahed, Cavity solitons, In Without Bounds: A Scientific Canvas of Nonlinearity and Complex Dynamics, Springer, 2013, 395-404. 